\section{Receptor isolation}

GPRALSI fwd

GPRALS1rev

GPRALS2fwd

GPRALS2rev

\section{q-PCR}

$S 7$ fwd

$S 7$ rev

$M C$ fwd

$M C$ rev

Vtgfwd

Vtgrev

$C P$ fwd

CPrev

GPRALSI fwd

GPRALSIrev

GPRALS2fwd

GPRALS2rev

$A S T-A$ fwd

AST-Arev
5'-3'

atgacgaacgagtctaaaatta

tcataaggggtccgcgttcga

atggtaagcgagaactacacgc

ttataaaatgtctggacagctg

cattctgcccaaaccgatgcgt

cgggaataccagatcctccagg

cetgcccacgacggtcatttactt

cgtcccgecctttccttctttg

acgaaaaccatgaccgctct

cttgggacggatcaccaaat

cgttctcggaggttgagac

ctgcgagtacgagtggaacg

ggttgccgcgaaccagag

ccaatactctccgaagggc

gtccaacccacagatgcg

gacgctcttgcaccatagc

gctacatcattgaggacgtgcg

atcagtccatcgtactcgtaat
$\mathrm{T}^{0} \mathrm{C} \quad$ Efficiency $\left(\mathrm{r}^{2}\right)$

60

60

64

$102 \%\left(\mathrm{r}^{2}=0.99\right)$

60

$103 \%\left(\mathrm{r}^{2}=0.99\right)$

$103 \%\left(r^{2}=0.99\right)$

60

$99.5 \%\left(r^{2}=0.99\right)$

60

$106 \%\left(r^{2}=0.99\right)$

56

$84 \%\left(r^{2}=0.95\right)$

58

$104 \%\left(r^{2}=0.99\right)$ 